Proceedings of the Conference of the Physics of Opto-Electronic Materials, 1970 General Motors $\mathrm{R}$ esearch Symposium held in Warren, Michigan October 5-6, 1970
UCR L-20358

Preprint

$\operatorname{Con} f-701026-1$

\title{
NONLINEAR OPTICAL EFFECTS
}

Y. R. Shen

October 1970

AEC Contract No. W-7405-eng-48
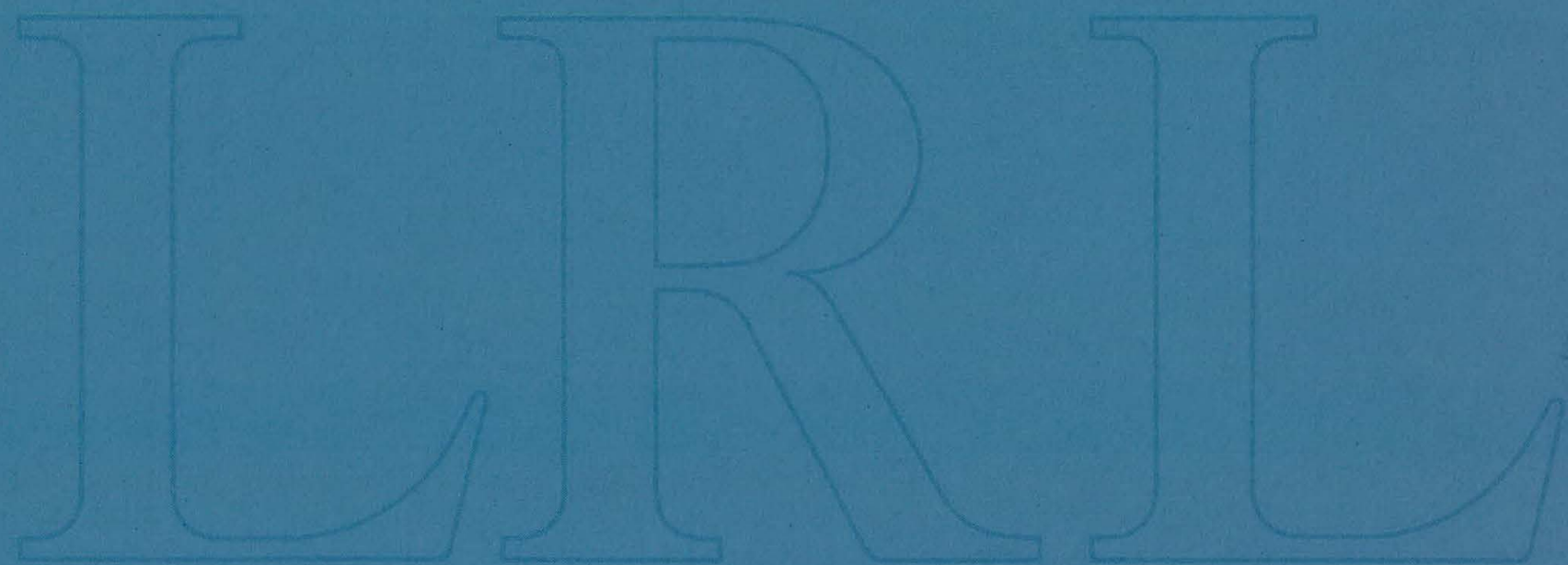

\section{LAWRENGE RADIATION LABORATORY UNIVERSITY of CALIFORNIA BERKEIEY}




\section{DISCLAIMER}

This report was prepared as an account of work sponsored by an agency of the United States Government. Neither the United States Government nor any agency Thereof, nor any of their employees, makes any warranty, express or implied, or assumes any legal liability or responsibility for the accuracy, completeness, or usefulness of any information, apparatus, product, or process disclosed, or represents that its use would not infringe privately owned rights. Reference herein to any specific commercial product, process, or service by trade name, trademark, manufacturer, or otherwise does not necessarily constitute or imply its endorsement, recommendation, or favoring by the United States Government or any agency thereof. The views and opinions of authors expressed herein do not necessarily state or reflect those of the United States Government or any agency thereof. 


\section{DISCLAIMER}

Portions of this document may be illegible in electronic image products. Images are produced from the best available original document. 


\author{
Nonilinear Optical Effects \\ Y. R. Shen \\ Department of Physics, University of California \\ and \\ Inorganic Materials Research Division, \\ Lawrence Radiation Laboratory, \\ Berkeley, California 94720
}

\begin{abstract}
Third-harmonic generation in cholesteric liquid crystals, far-infrared generation by ultrashort pulses, and self-focusing of light in nonlinear media are taken as examples for illustration of nonlinear optical effects. Depenting on the problems, different approximations are used in solving the nonlinear wave equations. Connection between nonlinear optical effects and properties of the media is briefly discussed.
\end{abstract} This report we United States Government. Nnergy sponsored by the nor the United States Atomic Energ the United States nor the their employees, nor any of Commission, nor any of thactors, or their employees, their contractors, subcontractors, or the or assumes any their cony warranty, express or implied, accuracy, comlegal liability or responsibility for the accion, apparatus, pleteness or usefulness of any ir reprcsents that its use product or process disclosed, or repights.

would not infringe privately owned 


\section{INTRODUCTION}

By definition, nonlinear optics deals with problems involving nonlinear interaction of light with matter. ${ }^{l}$ It arises $b \geqslant c a u s e$ the response of a medium to the fields is generally nonlizear,. as indicated by the nonlinear constituitive equation $\underset{\sim}{D}=\underset{\approx}{\varepsilon(\underset{\sim}{E})} \cdot \underset{\sim}{\mathrm{E}}$. Only when the field dependence in the dielectric tensor $\dot{\varepsilon}(E)$ is negligible, can the medium be approximated as linear. If, however, the fields are sufficiently strong, then the field dependence in $\varepsilon$ can no longer be neglected. The nonlinear response of the medium changes drastically the characteristics of wave propagation in the medium, and gives rise to many interesting nonlinear optical phenomena.

As an example, suppose we can expand $\underset{\approx}{(E)}$ into a power series of $\underset{\sim}{\mathbb{E}}$.

$$
\underset{\sim}{\varepsilon(E)}=\varepsilon_{0}+\varepsilon^{(2)}: \underset{\sim}{E}+\cdots
$$

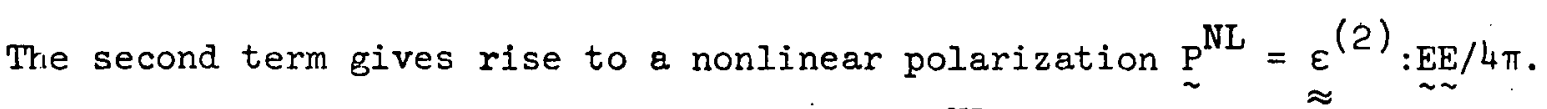
If $\underset{F}{\mathrm{i}}$ is oscilllating at a frequency $\omega$, then $\underset{\sim}{\mathrm{NL}}$ is oscillating at a frequency $2 \omega$. Since oscillating dipoles radiate, the nonlinear polarization $\mathrm{P}^{\mathrm{NL}}$ now acts as a source for the generation of the second-harmonic. field at $2 \omega$. Usually in the visible, $\varepsilon_{0}$ is around 3 esu, and $\varepsilon^{(2)}$ raneer from $10^{-4}-10^{-7}$ exu. For an 1 mput bean ol 1 Mwalts $/ \mathrm{cm}^{2}$ corresponding to $E \approx 20 \mathrm{esu}$, the second-harmonic generated from a $1-\mathrm{cm}$ $\mathrm{LiNbO}_{3}$ crystal $\left(\varepsilon_{\mathrm{zxx}}^{(2)} \approx 9 \times 10^{-6}\right.$ esu) can have a maximum intensity uround $100 \mathrm{Kwatts} / \mathrm{cm}^{2}$ which is certainly non-negligible.

Just as in linear optics, there are two types of problems in nonlinear optics. One is to relate the nonlinear optical constants to 
the microscopic properties of a given medium. Because of the higherrank tensors, a nonlinear dielectric tensor usually contains more independent elements than the linear one. We can, at least in principle; learn more about the properties of the medium by studying the nonlinear case. For this type of problems, what we often do is to measure the nonlinear optical constants on the one hand and, on the other hand, to calculate them from some microscopic theory. By comparing the theoretical and experimental results, we hope to learn something about the properties of the medium. Recently, there has been considerable interest in developing simple microscopic theories for evaluating lower-order nonlinear optical constants. In this respect, the chemical bond theory developed by Phillips, Van Vechten, Levine, etc. ${ }^{2}$ has turned out to be most successful. Here, since we already have several papers on the subject in the conference proceedings, we shall not dwell on it further.

The other type of problems in nonlinear optics is to study how the light waves propagate in a nonlinear medium with given nonlinear optical constants. This type of problem is basically more important. For example, in order to deduce the nonlinear optical constants of a medium from measurements, one must first understand nonlinear wave propagation in such a medium. In principle, these problems are fairly simple. All we have to do is to solve the nonlinear wave equations. Unfortunately, nonlinear wave equations are generally difficult to solve. Specific approximations must be used for specific problems. Physles detually comes in when we. try to use various approximations to find a solution. In this paper, we shall give three exarples to 
illustrate the point. These are the problems. which we are presently working on in our laboratory. Therefore, they also serve as examples for a section of the current nonlinear optical research.

II. PHASE-MATCHED THIRD-HARMONIC GENERATION IN LIQUID CRYSTALS

Harmonic generation in a nonlinear medium is one of the oldest problems in nonlinear optics. Phase-matched third-harmonic generation has been achieved in anisotropic crystals ${ }^{3}$ and in dye solutions. 4 Here, we would like to shor that it can also be achieved in cholesteric liquid crystals which have some unusual characteristics. 5

For convenience in later discussion, let us first review briefly the general characteristics of a cholesteric liquid crystal. 6 A cholesteric liquid crystal is usually composed of rod-like molecules. Fig. I shows the arrangement of molecules in such a medium. In each plane perpendicular to the $\mathrm{z}$-axis, the molecules are all aligned with the principal molecular axes along $\hat{\xi}$, and $\hat{n}$. As the plane advances along the z-axis, the direction of molecular alignment gradually rotates, so that the medium acquires an overall helical structure. Referred to the coordinates $\hat{x}$ and $\hat{y}$ in the lab system, the molecular axes $\hat{\xi}$ and $\hat{n}$ can be written as

$$
\begin{aligned}
& \hat{\xi}=\hat{x} \cos (2 \pi z / p)+\hat{y} \sin (2 \pi z / p) \\
& \hat{n}=-\hat{x} \sin (2 \pi z / p)+\hat{y} \cos (2 \pi z / p)
\end{aligned}
$$

where $p$ is the helical pitch, which can be varied by varying temperature, composition, external fields, etc. Because of its helical structure, a 
cholesteric liquid crystal has some interesting optical properties. It behaves like a grating in diffracting light and possesses strong optical activity. Since the pitch is comparable wi.th optical wavelengths, the macroscopic optical dielectric constants now vary periodically along $z$. In each plane of molecular alignment, the dielectric constants $\varepsilon_{\xi}$ and $\varepsilon_{n}$ along $\hat{\xi}$ and $\hat{n}$ respectively are clearly different. As a result of these characteristic properties, we have found some unusual phase-matching conditions for third-harmonic generation in a cholesteric liquid crystal as we shall see.

From the Maxwell equations, we can easily derive the nonlinear wave equation for generation of the third-harmonic field ${\underset{\sim}{E}}^{(3 \omega)} \exp (-i 3 \omega t)$ in a cholesteric liquid crystal.

$$
\begin{aligned}
{\left[\nabla x(\nabla x)-(3 \omega / c)^{2}\right.} & \varepsilon(z)] E^{(3 \omega)}=(3 \omega / c)^{2} 4 \pi \underset{\sim N L}{P}(3 \omega)
\end{aligned}
$$

where the nonlinear polarization $\mathrm{P}_{\sim \mathrm{NL}}(3 \omega)$ is given by

$$
4 \pi \underset{\sim N L}{P}(3 \omega)=\underset{\approx}{\varepsilon}(3)(z):{\underset{\sim}{E}}^{(\omega)} \underset{\sim}{(\omega)} \underset{\sim}{(\omega)}
$$

Because of the helical structure, both $\varepsilon(z)$ and $\varepsilon^{(3)}(z)$ are periodic functions of $\mathrm{z}$. We assume that the beam is collimated and is propagating along $\hat{z}$. Since the beam diameter is much larger than the optical wavelength, $\lambda$, in this case, the diffraction effect is negligible. Therefore, we can use the plane-wave approximation with $\nabla x(\nabla x)$ in Eu. (3) replaced by $-\partial^{2} / \partial z^{2}$. The resulting equation is most easily 


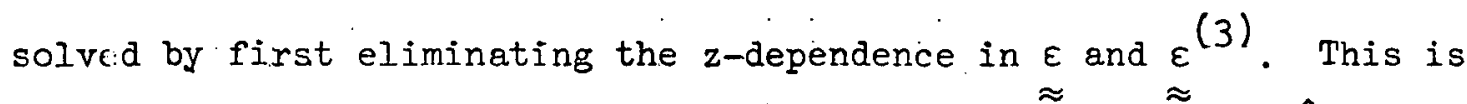
done by transforming to a coordinate system rotating around $\mathrm{z}$ with $\mathrm{a}$ rotational transformation $\mathrm{R}(\theta=2 \pi z / p) .5,7$ Physically, the helical struiture can be considered as a twisted birefringent medium, and the rotasional transformation is designed to untwist the helical structure.

In this rotating courdinate system, Eq. (3) now becomes

$$
\begin{aligned}
& {\left[\frac{\partial^{2}}{\partial z^{2}}-\left(\frac{2 \pi}{p}\right)^{2}+\left(\frac{4 \pi}{p}\right) \sigma \frac{\partial}{\partial z}+\left(\frac{3 \omega}{c}\right)^{2} \varepsilon_{\approx T}\right] F_{1 ! !}(3 \omega)} \\
& =-\left(\frac{3 \omega}{c}\right)^{2} \underset{\approx T}{\varepsilon_{T}}(3):{\underset{\sim T}{T}}^{E_{T}}(\omega) \underset{\sim T}{E_{T}}(\omega) \underset{E_{T}}{(\omega)}
\end{aligned}
$$

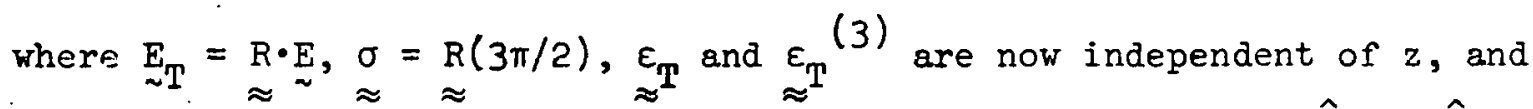
$\varepsilon_{\mathrm{T}}$ 1s diagonalized with the principal values $\varepsilon_{\xi}$ and $\varepsilon_{\eta}$ along $\hat{\xi}$ and $\hat{\eta}$ respectively. Consider first the Iinear wave propagation in the medium. The solution can be obtained by neglecting the nonlinear term in the wave equation, and is in the form ${ }^{\top}$

$$
\underset{\sim T}{E_{T}}(\omega)=\sum_{j= \pm} \mathbb{\&}_{\mathrm{Tj}}^{(\omega)} \exp \left[i \kappa_{j} z-i \omega t\right]
$$

with $\quad \&_{\sim T Y}(\omega)=\left(\hat{\xi} \&_{\xi}+\hat{n} \&_{n}\right)$

$$
\begin{aligned}
& \left|\kappa_{ \pm}{ }^{(\omega)}\right|=(\omega / c)\left\{\left(\varepsilon+\lambda^{\prime}\right)^{2} \pm\left[\left(\varepsilon+\lambda^{\prime}\right)^{2}-\left(\varepsilon-\lambda^{\prime}\right)^{2}+\alpha^{2}\right]^{1 / 2}\right\}^{1 / 2} \\
& \left(\varepsilon_{n}{ }^{(\omega)} / \varepsilon_{\xi}{ }^{(\omega)}\right)=i f_{j}{ }^{(\omega)}=i\left[k_{j}{ }^{2}+(\omega / c)^{2}\left(\lambda^{\prime}+\alpha-\varepsilon\right)\right] / 2 k_{j}(\omega / c) \lambda^{\prime}
\end{aligned}
$$

where $\dot{\varepsilon} \equiv\left(\varepsilon_{\xi}+\varepsilon_{n}\right) / 2, \alpha \equiv\left(\varepsilon_{\xi}-\varepsilon_{\eta}\right) / 2, \lambda^{\prime} \equiv \lambda / p, k_{-}$is negative for $\lambda^{\prime 2}>\varepsilon_{\xi}$ 
and $\varepsilon_{\sim \mathrm{T} j}^{(\omega)}$ is independent of $z$.

If the nonlinear term becomes non-negligible, there is then a continuous transfer of energy from the fundamental to the thirdharmonics through nonlinear coupling. As a result, the field amplitudes $\varepsilon^{(\omega)}$ and $\varepsilon^{(3 \omega)}$ should now depend on $z$. However, physically, since the nonlinear coupling is weak, we expect that the rate of energy. transfer is small, and hence

$$
\left|\partial \varepsilon_{T}^{(3 \omega)} / \partial z\right| \ll\left|\kappa^{(3 \omega)_{\varepsilon}}(3 \omega)\right| \text { and }\left|\partial^{2} \varepsilon_{T}^{(3 \omega)} / \partial z^{2}\right| \ll\left|\kappa^{(3 \omega)} \partial \varepsilon_{T}^{(3 \omega)} / \partial z\right| \text {. }
$$

Equation (5) can therefore be approximated by

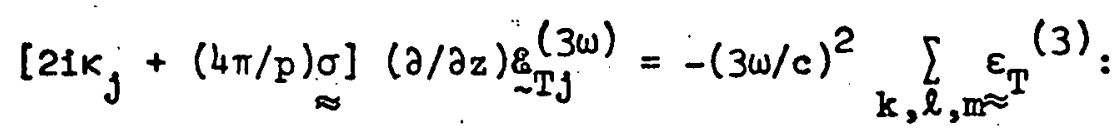

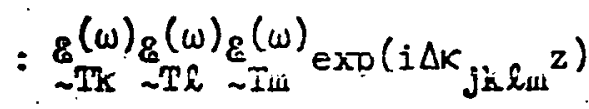

where $\Delta k_{j k \ell m}=k_{k}^{(\omega)}+k_{l}^{(\omega)}+k_{m}^{(\omega)}-k_{j}^{(3 w)}$.

Usually, the depletion of the fundamental pump energy by third-harmonic generation is also small. As a good approximation, the fundamental field amplitude $\varepsilon_{-T}^{(\omega)}$ can be taken as a constant. It is then easy to solve Eq. (7). We find, ${ }^{5}$ assuming $E_{T}^{(3 \omega)}=0$ at $z=0$;

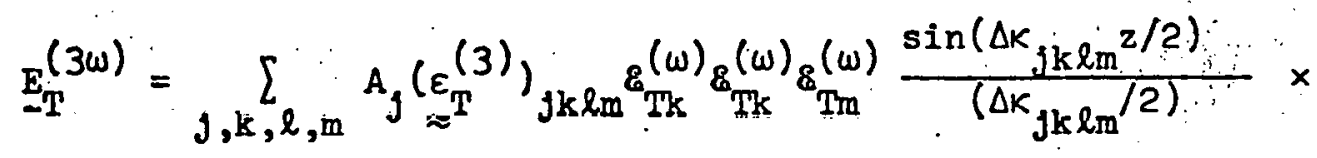

$$
\begin{aligned}
& x \exp \left[1\left(k_{j}^{(3 \omega)}+i \Delta k_{j k \ell m} / 2\right) z\right]
\end{aligned}
$$




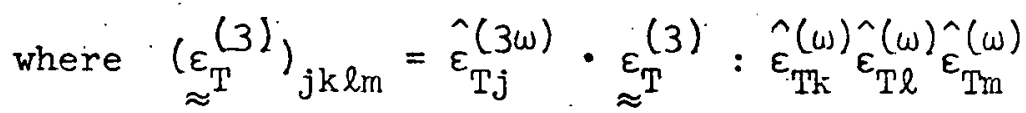

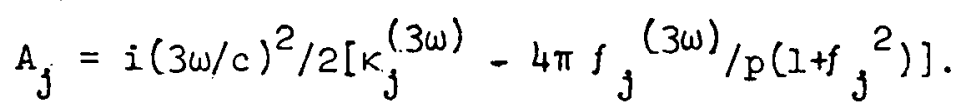

It is clear from the above equation that the third-harmonic intensity will be a maximum if the momentum or phase matching condition $\Delta k_{j k l m}=0$ is satisfied. This is possible in a cholesteric liquid crystal for several combintations of $j, k, l$, and $m$, since $k_{ \pm}$in $E q .(6)$ can be varied by varying the helical pitch p. Near phase-matching, $\Delta k_{j k \ell m} \approx 0$, the generated third-harmonic power is given by $(c / 2 \pi n) \int|\underset{\sim}{E}(3 \omega)|^{2} d x d y$, where

$$
\begin{aligned}
|\underset{\sim}{E}(3 \omega)|^{2} & =\left|\underset{\sim T}{E_{T}}(3 \omega)\right|^{2} \\
& \cong\left|A_{j}\left(\varepsilon_{T}^{(3)}\right)_{j k \ell m} E_{T k}^{(\omega)} E_{T \ell}^{(\omega)} E_{T m}^{(\omega)}\right|^{2} \frac{\sin ^{2}\left[\Delta k_{j k \ell m} z / 2\right]}{\left(\Delta k_{j k \ell m} / 2\right)^{2}} .
\end{aligned}
$$

If $\varepsilon_{T}^{(3)}$ for the medium is known, we can predict the third-harmonic power generated at phase-matching by a given input beam. Conversely, measurements of the third-harmonic output near phase-matching should, in principle, enable us to determine the nonlinear dielectric constant $\left(\varepsilon_{\mathrm{T}}^{(3)}\right)_{j \mathrm{k} \ell \mathrm{m}}$ from $\mathrm{Eq}$. (9).

The experimental setup for harmonis berration experiments is fairly standard. 8 In our case, a mode-locked Nd-glass laser beam was normally incident on a liquid crystal sample whose helical axis was made parallel to the direction of beam propagation. The third-harmonic output was then detected by a photomultiplier with proper filtering. In order to achieve phase-matching, the helical pitch of the sample was 
tuned by adjusting the sample temperature. We have observed in our experiments most of the predicted phase-matching conditions. ${ }^{9}$ Here, we shall mention only two of them.

In the first case, a cholesteric mixture composed of 1.75 parts of cholesteryl chloride and 1 part of cholesteryl myristate was used. 5 Knowing $\varepsilon$ and $\alpha$ in $E q$. (6) from measurements, we calculated $k_{ \pm}$as functions of the pitch $\mathrm{p}$. We then predicted that the phase-matching condition $\Delta k_{++++}=0$ can be satisfied with $p= \pm 17.3 \mu \mathrm{m}$ where + and refer to right and left helexities. The sample temperatures corresponding to these pitch values are 49.4 and $54.2^{\circ} \mathrm{C}$ respectively. The thirdharmonic waves in this case propagate in the same direction as the fundamental waves. In Fig. 2 , the observed third-harmonic intensity is plotted as a function of temperature. It is seen that the phasematching peaks indeed appear at the predicted temperatures.

In the second case, a mixture of $30 \%$ cholesteryl chloride, $35 \%$ cholesteryl nonanoate, and 35\% cholesteryl oleyl carbonate was used. 9 The phase-matching condition $\Delta K \ldots=0$ can now be satisfied with $p=0.47 \mu \mathrm{m}$ at a sample temperature of $38.2^{\circ} \mathrm{C}$. However, in this case, $\kappa_{-}^{(\omega)}$ and $\kappa_{-}^{(3 \omega)}$ correspond to fundamental and third-harmonic waves propagating in opposite directions in the laboratory frame. The observed third-harmonic phase-matching peak again appeared at the predicted temperature, as shown in Fig. 2. Here, the observed peak is much broader than the theoretical one calculated under the assumption that the fundamental laser beam is monochrubatic. In the experiments, the mode-locked laser pulses had roughly a $10 \mathrm{~cm}^{-1}$ band of frequencies. 
Since different frequency components lead to slichtly different phase-matching temperatures here, the overall phase-matching curve was now appreciably broadened. One may have noticed that in this case, with the fundamental and the third-harmonic waves propagating in opposite directions, the linear momentum does not seem to be conserved in the laboratory frame. However, the momentum difference is actually comperisated by the lattice momentum. ${ }^{10}$ This is equivalent to an umflapp prooese in a cryctal, The unit lattice momentum here io given by $2 \pi / p$, as a result of the helical structure of the liquid crystals. III. FAR-INFRARED GENERATION BY ULTRASHORT PULSES IN NONLINEAR CRYSTALS Far-infrared radiation can be generated by the difference-frequency generation process in a nonlinear crystal. ${ }^{11}$ Two laser beams with two different frequencies can be used as the primary pumping fields. ${ }^{12}$ An ultrashort pulse, however, contains a broad band of frequencies. for example, a l-psec. pulse has a bandwidth of about $13 \mathrm{~cm}^{-1}$. In a nonlinear crystal, the different frequency components in the band can beat with one another to generate difference-frequency fields in the far-infrared. From the practical point of view, this nonlinear optical process has the potential of providing a powerful, far-infrared source.

The wave equation for the far-infrared field $\underset{\sim}{\mathrm{E}}$ generated by beating of the laser field $\underset{\sim}{\mathrm{E}}$ has the form

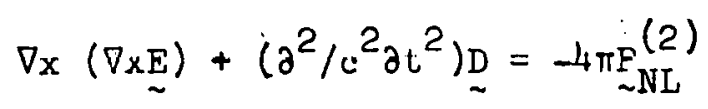

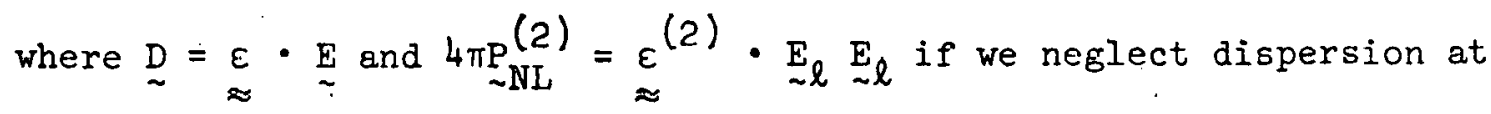


the far infrared. One would expect that the solution of the c.bove equation is quite similar to the case of harmonic generation. However, since the far-infrared frequencies are now comparable with the inverse of the ultrashort pulse width, we can no longer neglect the time-derivative of the field amplitude in the wave equation as. we did in the case of harmonic generation. The approximation of rep acing $\overrightarrow{\nabla x}(\nabla \times \underset{\sim}{x})$ by $-\left(\partial^{2} / \partial z^{2}\right) \underset{\sim}{E}$ also breaks down because the far-infrarted wavelengths are comparable with the transverse dimension of the beam. Diffraction of the far-infrared now becomes important, especially in the low-frequency limit. As a result, the wave vectors of th: farinfrared radiation generated in the nonlinear crystal span over a large cone. Because the refractive index at the far-infrared is usually large (6.6 for ordinary ray in $\left.\mathrm{LiNbO}_{3}\right)$ reflection and refraction at the boundaries are also important. For a crystal slab with the beam propagating perpendicular to the plane surfaces, the far infrared generated with a wave vector at a large angle is totally reflected at the boundary surfaces and can never get out of the crystal. The far infrared generated at small angles sees a strong Fabry-Perot interference. It also suffers strong refraction at the boundaries, so that the far-infrared radiation is spread into a broad, cone outside the crystal. We lhen realize that, in the present case, we must solve the complete 4-dinensional equation in (10) with proper boundary conditions. The calculation is clearly much more difficult than that for harmonic Eeneration. However, with appropriate approximations, we can greatly sillplify the solution.' Because of the linlted space here, we shall 
not go into the discussion of how we actually solve the equation. We have. calculated far-infrared spectra generated in $\mathrm{LiNbO}_{3}$ by $\mathrm{Nd}$ mode-locked pulses under different conditions. Figure 4 gives one example. 13

In Fig. 4, the far-infrared is generated in a $1 \mathrm{~mm}$ slab of $\mathrm{LiNbO}_{3}$ through the dielectric constant $\varepsilon_{z z z}^{(2)}$ by pulses with a 2-psec. pulse width. This spectrum is quite easy lo urderstand physically. The dashed curve is calculated without boundary conditions. The three pcakc at $5 ; 8.1$, and $11.8 \mathrm{~cm}^{-1}$ come in $8 \mathrm{~s}$ the seroniary peaks of the phase-matching curve with phase matching occurring at zero frequency. They correspond to $\Delta \mathrm{k}=3 \pi / 2,5 \pi / 2$, and $7 \pi / 2$ respectively, where $\Delta \mathrm{k}$ is the momentum mismatch in the difference-frequency generation process. There would be the major phase-matching peak at zero frequency, if it were not for the fact that low-frequency fields do not radiate efficiently. The cutoff of the phase-matching curve at the lowfrequency end due to radiation and diffraction effects gives rise to the first peak at $2 \mathrm{~cm}^{-1}$. in Fig. 4. With the boundary conditions included, the curve is now modified by the Fabry-Perot interference pattern, as shown by the solid curve with spikes. However, in actual experiments, the spectrometer has limited resolution. If the resolution is larger than the width of the spikes, then after convoluting the solid curve in Fig. 4 with the slit function, the spectrum would again have the form of the dashed curve. This has been shown to agree vell with the experimental results. 14 A Nd mode-locked laser generating pulses with peak power of a few Gwatts was used in the experiments. The spectrum of the far-infrared output was analyzed by a Michelson interferometer. 
Normalization against fluctuations was achieved by the usual method of splitting the far-infrared output into two beams. Details of the experiments will be published elsewhere. 14

Instead of $\varepsilon_{z z z}^{(2)}$, we can also use the dielectric constant $\varepsilon_{y y z}^{(2)}$ of $\mathrm{LiNbO}_{3}$ by properly orienting the crystal with respect to the laser polarization. Phase matching of the collinear difference-frequency generation. now occurs at different frequencies depending on the orientation of the crystal. Our calculations show that, in this case, the far-infrared spectra consist of sharp phase-matohing curves. The positions of the phase-matching peaks can be tuned by rotating the crystal. Experimental results are again in agreement with theoretical prediction.

IV. SELF-FOCUSING OF LIGHT IN LIQUIDS

As a last example, let us discuss how the propagation of a laser beam in a medium can be vastly different from the linear wave propagation because the refractive index of the medium depends on the laser intensity. This is one of the' most fascinating, but also the most difficult problems in nonlinear optics. Only after many years of struggle, we now begin to gain insight in the solution of the problem.

Let us first have a qualitative discussion on the self-focusing phenomenon. Consider an isotropic medium, such as a liquid, as an example. Since the medium has an inversion symmetry, its refractive index (or dielectric constant) can be written as

$$
n(E)=n_{0}+\Delta n
$$


where we have, in the steady-state,

$$
\Delta n=\Delta n_{0}=n_{2}|E|^{2}+n_{4}|E|^{4}+\cdots
$$

Physically, the induced refractive index $\Delta n$ arises because of molecular reorientation and redistribution, electrostriction, and electronics effects. In experiments with Q-switched laser pulses propagatir:g in liquids, the contribution from molecular reorientation often dominctes. ${ }^{15}$ The magnitude of $n_{2}$ ranges from $10^{-11}$ to $10^{-13}$ esu. In most media, the induced $\Delta \mathrm{n}$ is a positive quantity.

We now propagate a laser beam into such a medium. Assume that the collimated laser beam has inftially a plane wavefront, but has a finite cross-section with a Gaussian intensity profile. Then, as the beam propagates into the medium, the central part of the baam sees a larger refractive index than the edge, and therefore travels at a slower speed than the edge. As a resull, the wavefront of the bcam gete distorted more and more as the beam propagates (see Fig. 5). Since propagation of energy must be normal to the wavefront, the beam now appears focused by itself. The beam distortion, being a nonlinear, cumulative effect, makes the beam focus rather suddenly to a small spot of a few micruss in radius.

From the above description, it is seen that the finite beam cross-section is important for self-focusing, and in the focusing region, both the intensity and the phase of the optical field change rapidly, so that they cannot be considered as subject to small perturbation. The wave equation for this problem can be written as 


$$
\left[\nabla x \cdot(\nabla x)+\left(\partial^{2} / c^{2} \partial t^{2}\right)\left(n_{0}+\Delta n\right)^{2}\right] E=0
$$

where $\Delta n$, if it is mainly due to molecular orientation, can be assumed to obey a simple relaxation equation

$$
(\partial / \partial t+I / \tau) \Delta n(t)=\Delta n_{0} / \tau
$$

For ordinary, non-viscous liquids, the orientational relaxation time $\tau$ is of the order of a few picoseconds. The solution of the above equations, being extremely nonlinear, is clearly very difficult. In order to facilitate the solution by approximations, we must refy very much on our physical judgment.

When the laser pulse is much longer than the relaxation time, the time derivative in $\mathrm{Eq}$. (13) can be neglected. If the beam intensity is not exceptionally high, then we can write

$$
\Delta n(t) \cong n_{\partial}|E(t)|^{2}
$$

Assuming a steady-state case with the above nonlinearity, Kelley ${ }^{16}$ has found that a beam with power $P$ would self-focus to a point at

$$
\begin{aligned}
& z_{f} \cong K /\left(P^{1 / 2}-\ddot{P}_{c r}^{1 / 2}\right) \\
& K=(n / 4)\left(a^{2} / f\right)\left(c / n_{2}\right)^{1 / 2}
\end{aligned}
$$


where $P_{c r}$ is the critical power for self-trapping, 17 a is the bear radius, and $f$ is a parameter of the order of $1 .^{18^{\prime}}$ As seen from Eq. (14), there is a critical power $P_{c r}$ below which the beam cannot self-focus $\left(z_{f}<0\right)$. This happens because diffraction overcomes the self-focusing action. Eq. (14) has actually been verified by experiments. 18

However, for a pulsed laser beam, the input power $P$ is a function of time. Consequently, from Eq. $(14), z_{f}$ should also be a function of time, and hence, the focal spot should appear moving along the axis. 1.9 If we take a time-integrated photograph of the self-focused beam from the side, the continuous series of focal spots would then appear as a bright filament. This is the so-called small-scale filament many research groups have observed. 20

Let us see more quantitatively how the focal spot moves along the axis. Suppose we have a $\quad 1$-nsec. input pulse with a given pulse shipe as shown in Flg. 6. At $t_{A}$, the 11 ght beam with a power $P\left(t_{A}\right)$ enters the medium. It then propagates in the medium with the light velocity $c / n_{0}$ (along the dashed line in Fig. 6), and finally selffocuses at $z_{A}$. At an earlier time, the light beam entering the medium has a smaller power, and it therefore self-focuses at a distance farther away. With the help of Eq. (14), we can now plot out the whole curve 
describing the position of the focal spot as a function of time. ${ }^{21}$

If $\ell$ is the length of the medium, the focal spot should first appear inside the medium at $z_{D}$. It then immediately splits into two. One first moves backward towards the laser to $z_{B}$ and then turns around and moves forward. The other simply moves all the way to the end of the medium. Here, let us consider only the second branch since it is more interesting, although experiments have proved the existence of both branches. $^{21-23}$ As shown in Fig. 5, the forward-moving branch has a slope larger than $c / n_{0}$, and therefore the focal spot must have a velocity faster than the velocity of light in the medium. This can be demonstrated quite easily by experiments. We can use liquid as the nonlinear medium, and immerse two beam splitters in it. Each beam splitter couples out a short light pulse when the focal spot strikes the beam splitter. Knowing the distance between the two beam splitters, we can find, by measuring the time separation between the two pulses, the average velocity for the focal spot to travel from one beam splitter to the other. This was actually done. ${ }^{23}$ In one cast, for example, the two beam splitters immersed in toluene were separated by $15 \mathrm{~cm}$. It would take 0.75 nsec. for light to travel such a distance, but we measured a time of 0.25 nsec, between the two pulses. That the focal spot can move faster than light does not violate the law of relativity, since the continuous series of focal spots is actually formed by continuous focusing of different parts of the inpul pulse. A direct consequence of the fact that the rocal spot moves faster than light is: the possible formation of trapped filaments of light. ${ }^{23}$. Because of its 
high intensity, the moving focal spot leaves a track of induced $\Delta \mathrm{n}$, which lasts over a period of the order of the relaxation time $\tau$. This channel of $\Delta \mathrm{n}$ forms an optical, waveguide traveling along with the focal spot. The self-focused light trailing after the forward-moving focal spot can then be partially trapped in the waveguide for a certain distunce. The trapping lcngth depends on the relative velocity of the focal spot with respect to light. If the focal spot has a velocity close to the light velocity, so that it keeps in step with the light for a long distance, then a long trapped filament can result. Since the trapping length varies with time as a result of the focal-spot movement, light emitted from the filament at the end of the medium is strongly phase-modulated, and hence shows a remarkable spectral broadening with semi-periodical structure (a broadening of a $\mathrm{fcw}$ hundred to a few thousand $\mathrm{cm}^{-1}$ against a lincwidth of less than $1 \mathrm{~cm}^{-1}$ for the input laser beam). ${ }^{24}$. The model is also successful in explaining many snomalous observations on small-scale filaments. ${ }^{23}$ However, we shall not go into detailed discussion here.

\section{v. CONCLUSION}

The above three examples illustrate some general asperts of nonlinear optical research. There are, of course, many other interesting nonlinear optical problems, such as stimulated light scattering, nonlinear propagation of ultrashort pulses, self-induced transparency effect, etc. The field of nonlinear optics is still. growing. So far, research on nonlirear optics has been mainly on investigation of basis phenomena. However, quite a few important applications of nonlinear optics have 
already been found. The coherent, tunable light source is just one example. It is hopeful that someday we may find nonlinear optics at least as useful as linear optics both in practical applications and in studying properties of matter.

\section{ACKNOWLEDGEMENTS}

This work was performed under the auspices of the U. S. Atomic Energy Commission. 


\section{REFERENCES}

1. For detailed discussion see, for example, N. Bloembergen, Nonlinear Optics (W. A. Benjamin, Inc., New York, 1965).

2. J. C. Phillips, Phys. Rev: Letters 20, 550 (1968).

J. C. Phillips and J. A. Van Vechten, Phys. Rev. 183, 709 (1969).

J. A. Van Vechten, Phys. Rev. 182, 891; 187, 1007 (1969).

B. F. Trevine, Phys, Rev. Letters 22, 787 (1969); 25, 440 (1970).

3. P. D. Maker and R. W. Terhune, Phys. Rev. 137, A801 (1965).

4. P. P. Bey, J. F. Giuliani, and H. Rabin, Phys. Kev. Letter's 19, $819(1967)$.

5. J. W. Shelton and Y. R. Shen, Phys. Rev. Letters 25, 23 (1970).

6. See, for example, G. W. Gray, Molecular Structure and the Properties of Liquid Crystals (Academic Press, New York, 1962) p. 39.

7. H. N. de Vries, Acta Cryst. L, 219 (1951).

8. See, for example, R. K. Chang and L. K. Galbraith, Phys. Rev. 171, $993(1.968)$.

9. J. W. Shelton and Y. R. Shen (to be published), presented at the Sixth International Quantum Electronics Conference, Kyoto, Japan, 1970) paper $11-3$

10. N. Bloembergen (private communication).

11. F. Zernike and P. R. Berman, Phys. Rev. Letters 15, 999 (1963).

12. D. W. Faries, K. A. Gehring, P. L. Richards, anı Y. R. Shen, Phys. Rev. 180, 363 (1969).

N. Vun Trun eurd C. K. N. Patel, Phys. Rev. Tofters??, 463 (1969).

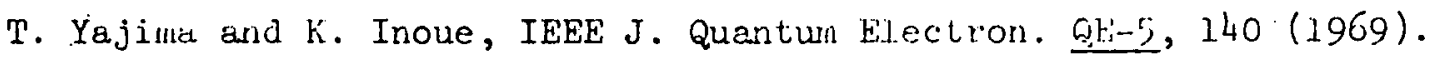

13. J. R. Morris and Y. R. Shen (to be published). 
14. K. H. Yang, Y. R. Shen; J. W. Shelton, and P. L. Richards, (to be published). A preliminary report was presented at the Sixth International Quantum Electronic Conference at Kyoto, Japan, 1970; paper 8-6.

15. Y. R. Shen, Phys. Letters 20, 378 (1966).

16. P. L. Kelley, Phys. Rev. Letters 15, 1005 (1965).

17. R. Y. Chiao, E. Garmire, and C. H. Townes, Phys. Rev. Letters 13, $479(1964)$.

18. C. C. Wang, Phys. Rev. Letters 16, 344 (1966).

19. A. L. Dyshko, V. N. Lugovoi, and A. M. Prokhorov, Zh. Eksperim.

i Theo. Fiz. Pis'ma Redakt. 6, 655 (1967), [Translation: JETP: Letters 6 , 146 (1967)]; V. N. Lugovoi and A. M. Prokhorov, ibid. I, 153 (1968) [Translation: JETP Letters I, 344 (1966).

20. R. Y. Chiao, E. Garmire, and C. H. Townes, Phys. Rev. Letters 13 , 479 (1964); R. Y. Chiao, M. A. Johnson, S. Krinsky, H. A. Smith, C. H. Townes, and E. Garmire IEEE J. Quantum Electron. QEE-2, 467 (1966); R. G. Brewer, J. R. Lifsitz, E. Garmire, R. Y. Chiao, and C. H. 'l'ownes, Phys. Rev. 166, 326 (1968).

21. M. M. T. Loy and Y. R. Shen, Phys. Rev. Letters 22, 994 (1969), 22. V. V. Korobkin, A. M. Prokhorov, R. V. Serov, and M. Ya. Shchelev, ZHETF. Pis. Red. 11, 1.53 (1970); [Translation: JETP Letters I], $94(1970)]$.

23. M. M. T. Loy and Y. R. Shen (to be published); presented at the Sixth International Quantum Electronic Conference, Kyoto, Japan 1970, paper 9-7. 
24. F'. Shimizu, Phvs. Rev. Letters 19, 1097 (1967);

R. G. Brewer, Phys. Rev. Letters 19, 8 (1967);

A. C. Cheung, D. M. Rank, R. Y. Chiao, and C. H. Townes, Phys. Fiev. Letters 20, 786 (1968). 
FIGURE CAPTIONS

Fig. 1. Arrangement of molecules in a cholesteric liquid crystal.

(Taken from I. G. Chistyakov, Usp. Fiz. Nauk 89, 563 (1966)

[Translation: Uspekhi 9, $551(1967)])$.

Fig. 2. Normalized third-harmonic intensity vs temperature near the phase matching temperatures for the mixture of 1.75 cholesteryl chloride and 1 cholesteryl myristate, in a cell $130 \mu \mathrm{m}$ thick. The peak at the lower temperature (corresponding to left helical structure) is generated by left-circularly polarized fundamental waves and the one at the higher temperature by right-circularly polarized fundamental waves. The solid line is the theoretical phase-matching curve and the dots are experimental data points. The uncertainty in the experimental third-harmonic intensity is about $20 \%$.

Fig. 3. Backward-propagating third-harmonic intensity vs temperature near the phase-matching temperature for the mixture of $30 \%$ cholesteryl chloride, $35 \%$ cholesteryl nonanoate, and $35 \%$ cholesteryl oleyl carbonate, in a cell of $130 \mu \mathrm{m}$ thick. The solid curve is the theoretical phase-matching curve assuming a monochromatic incoming field.

F'ig. 4. Calculated spectrum of the far-infrared generated by a 2-psec. short $\mathrm{Nd}$ laser pulse in a $1-\mathrm{mm} \mathrm{slab}$ of $\mathrm{LiNbO}_{3}$, oriented with the z-axis parallel both to the laser polarization and to the surfaces of the slab. The dashed and the solid curves were.calculated with and without the boundary conditions respectively. 
fig. 5. Description of self-focusing of a laser beam in a nonlinear mediun.

rig. 6. The lower trace describes the input power $P(t)$ as a function of time $t$. The peak power is 42.5 Kwatts and the half width at the $\mathrm{e}^{-1}$ point is 1 nsec. The upper trace, calculated from Eq. (1), describes the position of the focal spot as a function of time. The values of $P_{c r}$ and $K$ used are 8 Kwatts and $11.6 \mathrm{~cm} /(\mathrm{KW})^{1 . / 2}$ respectively, which correspond roughly to an input bean of $400 \mu$ in diameter propagating in $\mathrm{CS}_{2}$. The dotted lines, with the slope equal to the light velocity in $\mathrm{CS}_{2}$, indicate how the light propagates along the $z$-axis at various times. 

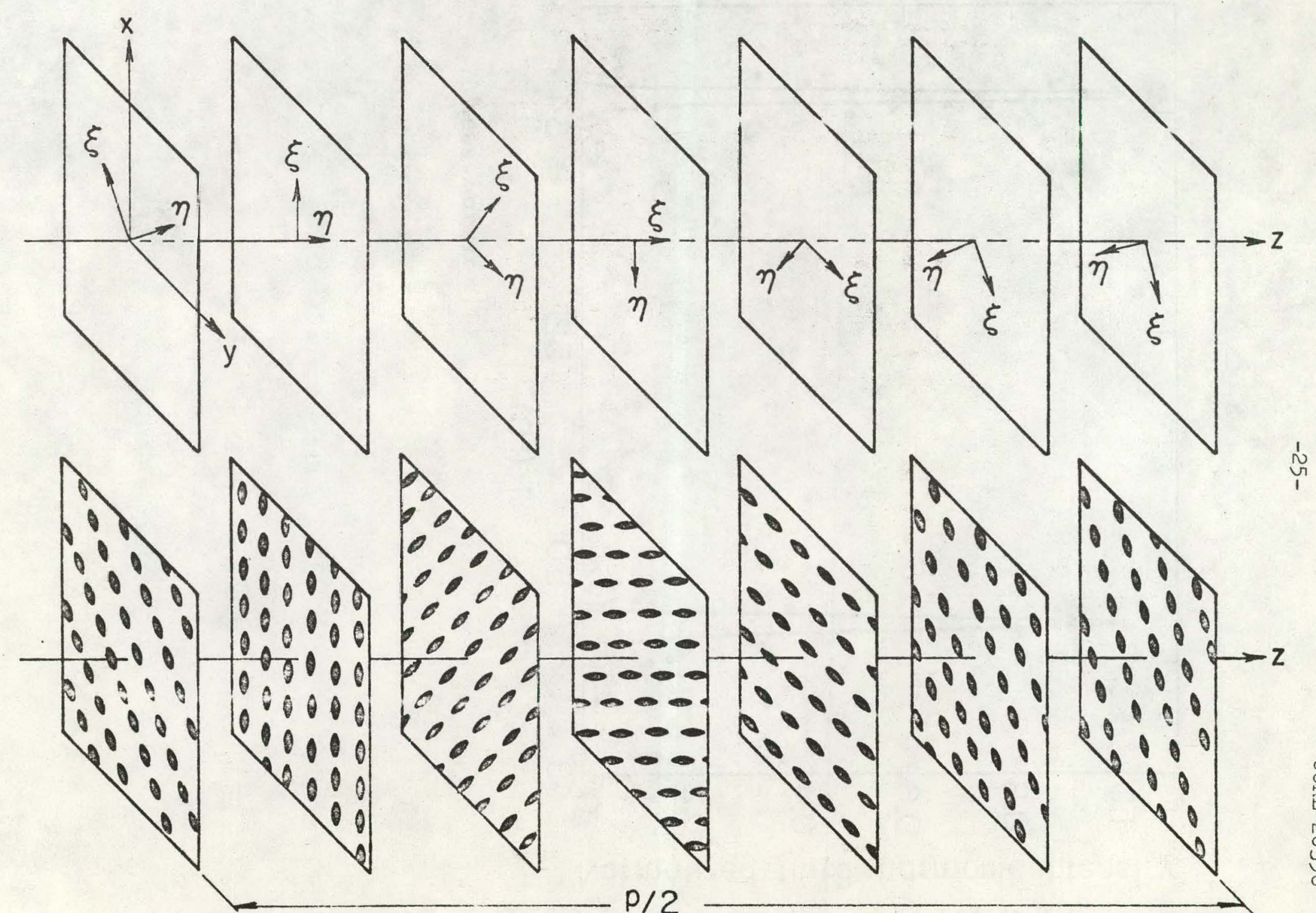

XBL 7010-6790 


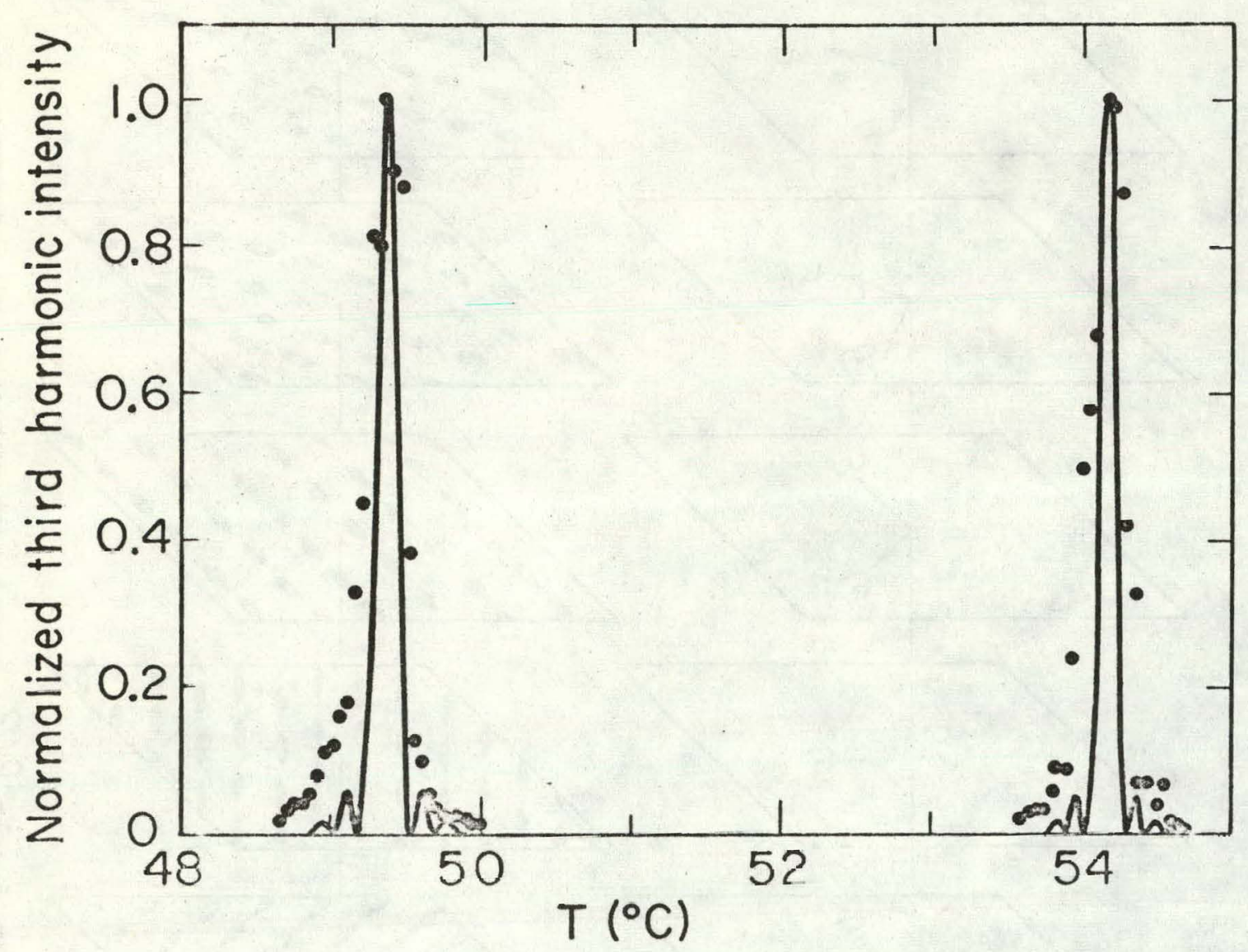

XBL $704-2692$

Fig. 2 


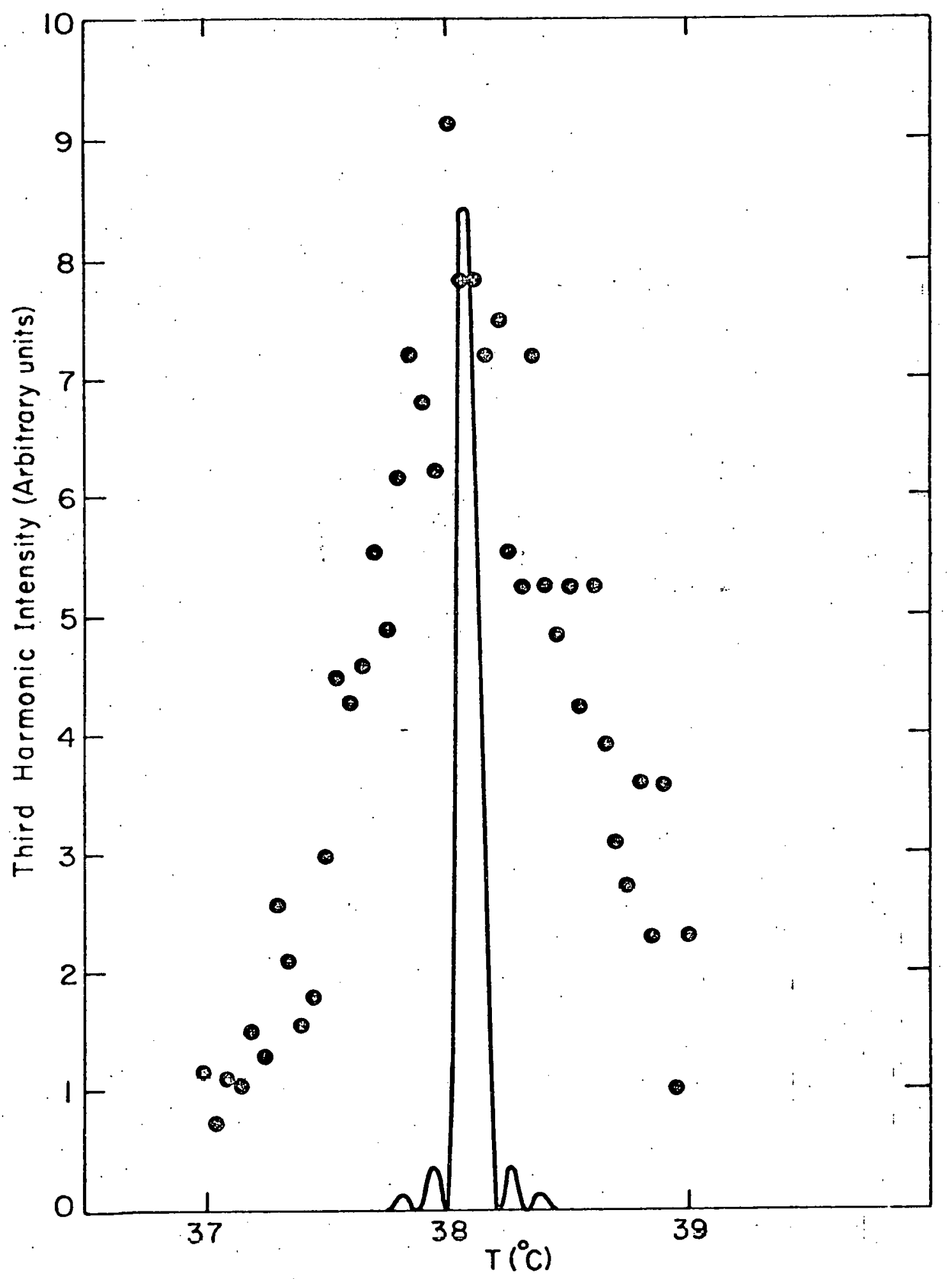

XBL $7010-1791$

Fig. 3 
$-28-$

UCRL-20358

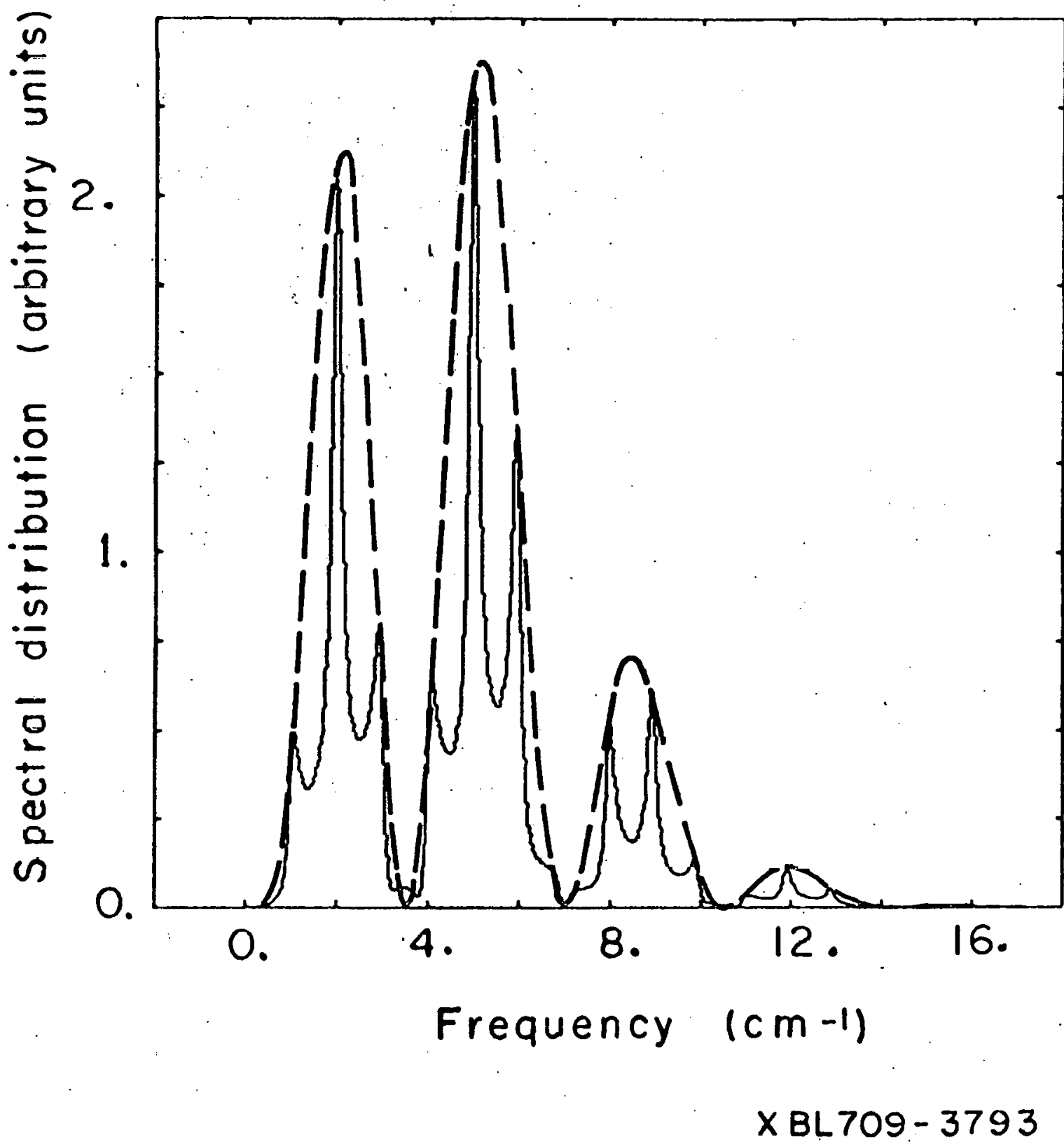

Fig. 4 

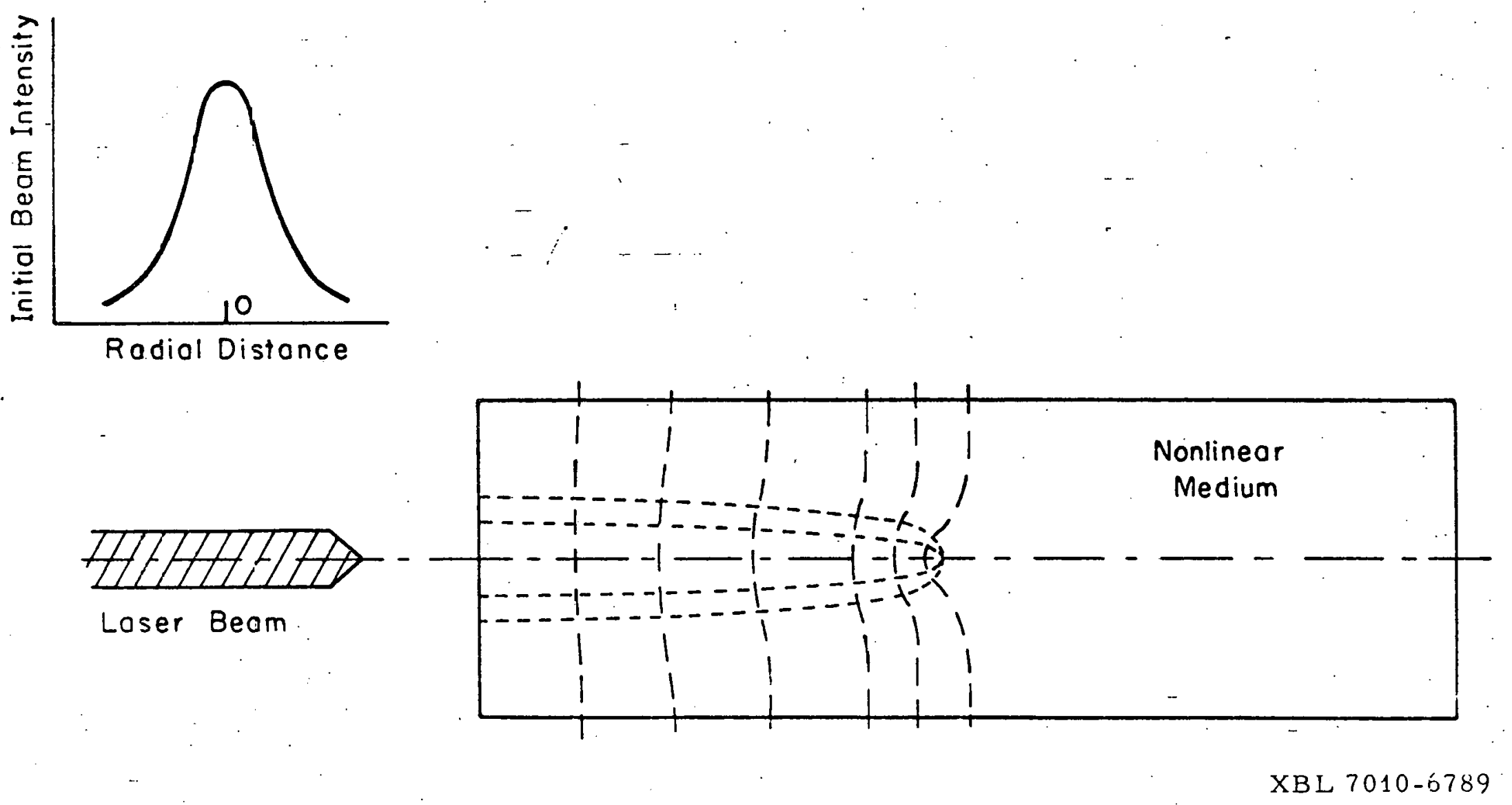

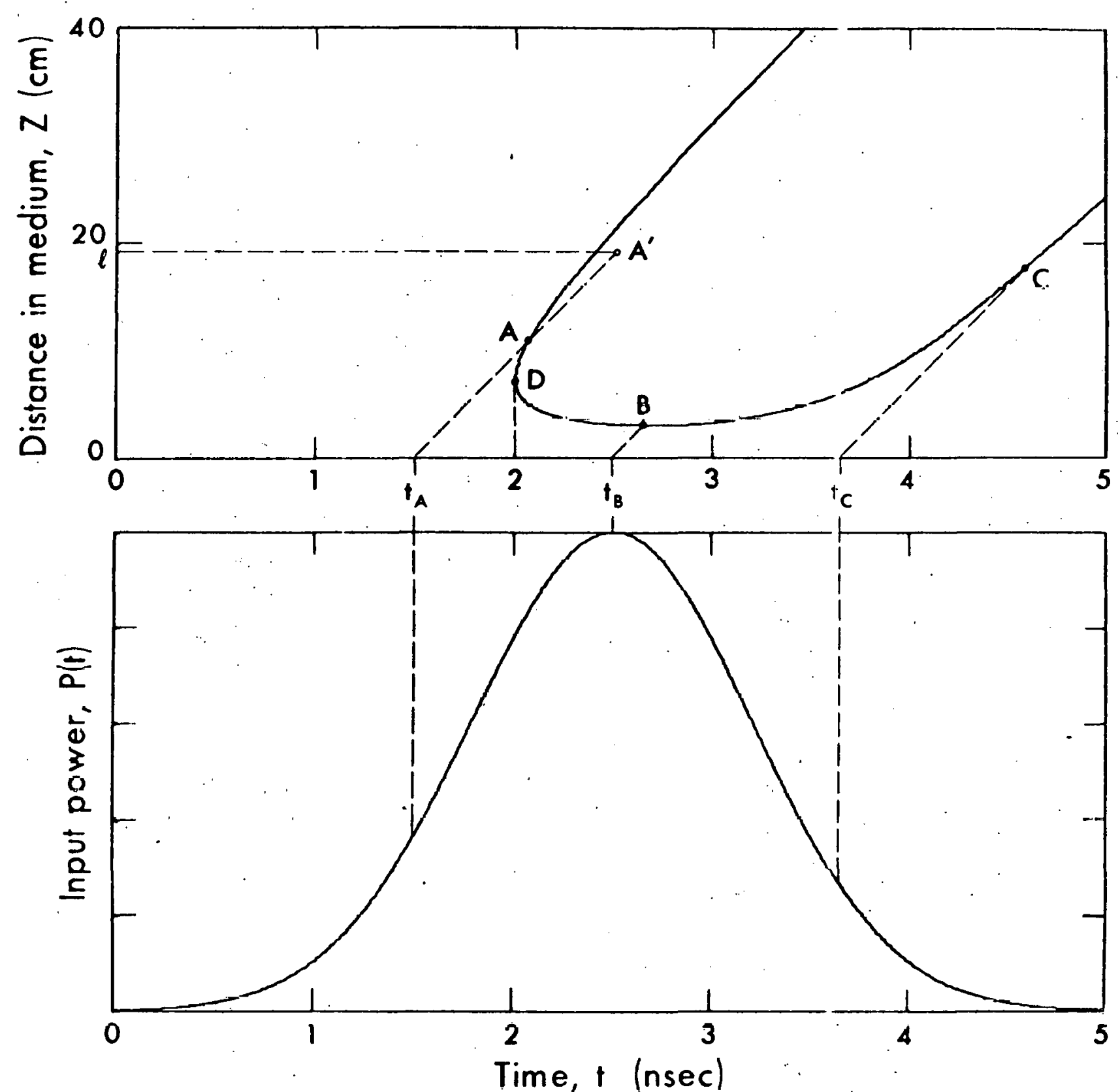

XBL 7010-6788

Fig. 6 


\section{LECAL NOTICE}

This report was prepared as an account of Government sponsored work. Neither the United States, nor the Commission, nor any person acting on behalf of the Commission:

A. Makes any warranty or representation, expressed or implied, with respect to the accuracy, completeness, or usefulness of the information contained in this report, or that the use of any information, apparatus, method, or process disclosed in this report may not infringe privately owned rights; or

B. Assumes any liabilities with respect to the use of, or for damages resulting from the use of any information, apparatus, method, or process disclosed in this report.

As used in the above, "person acting on behalf of the Commission" includes any employee or contractor of the Commission, or employee of such contractor, to the extent that such employee or contractor of the Commission, or employee of such contractor prepares, disseminates, or provides access to, any information pursuant to his employment or contract with the Commission, or his employment with such contractor. 
